55. Jahresversammlung der Schweizerischen Gesellschaft für Dermatologie und Venereologie 20. und 21. Oktober 1973 in Bern

Dermatologica, $1974 ; 148: 298$

\title{
Therapie der Gonorrhö mit Bactrim
}

F.

Gilliet

Dermatologische Universitätsklinik (Vorsteher: Prof. H. Storck), Zürich

Sonderdruckbestellungen an: Dr. F. Gilliet, Dermatologische Universitätsklinik Zürich, CH-8000 Zürich (Schweiz)

Zusammenfassung

Es werden die Resultate von Bactrim (Kombinationspräparat, enthaltend 400 mg Sul-

fomethoxazol und $80 \mathrm{mg}$ Trimethoprim pro Tablette) bei der Therapie der akuten Gonorrhö untersucht. Wie für Penizillin gilt auch für Bactrim, dass möglichst hoch dosiert werden sollte, jedoch sind zur Eindosentherapie bereits potentiell toxische Dosen nötig, so dass der Nachteil einer Mehrdosentherapie in Kauf genommen werden muss. Dabei konnte statistisch gezeigt werden, dass innerhalb der therapeutischen Breite die Resultate ver-schiedener Dosierungen gleich sind. Bei hoher Dosierung tritt der Erfolg jedoch schneller ein, die Behandlung wird kürzer, die nötige Gesamtdosis kleiner. Als geeignete, d.h. höchste ohne toxische Risiken tolerierbare Dosis zur Therapie der akuten Gonorrhö kön-nen 8 Tabletten täglich, verteilt auf zwei Gaben, während zwei Tagen, empfohlen werden. Eigene Untersuchungen, deren Resultate mit den aus der Literatur kollektiv errechneten übereinstimmen, ergeben eine Erfolgsquote von etwa 90\%. Ein gleichzeitig von uns durch-geführter Therapievergleich mit wässerigem Penizillin (Eindosentherapie mit 4 Millionen Einheiten Hydracillin forte) zeigte, dass die Bactrim-

Resultate gleichwertig sind.

Man kann Bactrim bei der Therapie der akuten Gonorrhö verwenden, wenn man eine perorale Mehrdosentherapie bei der Behandlung dieser Geschlechtskrankheit akzeptieren will. Es wird ersichtlich, dass der Eindosentherapie mit Penizillin bei der Theiapie der akuten Gonorrhö keine Vorzugsstellung mehr zukommt.

1 Erscheint in extenso in der Schweizerischen medizinischen Wochenschrift. 